\title{
Online Estimation of Soil Grain Diameter during Dredging of Hopper Dredger Using Continuous-Discrete Feedback Particle Filter
}

\author{
Zhen Su, ${ }^{1,2}$ Zhao-Xu Zhou, ${ }^{2}$ Meng-Hong Yu, ${ }^{2}$ Wei Yuan, ${ }^{2}$ and Jing-Qi Fu ${ }^{1 *}$ \\ ${ }^{1}$ Department of Automation, College of Mechatronic Engineering and Automation, \\ Shanghai University, Shanghai 200072, China \\ ${ }^{2}$ School of Electronics and Information, Jiangsu University of Science and Technology, Zhenjiang 212003, China
}

(Received November 23, 2018; accepted February 18, 2019)

Keywords: hopper dredge, soil grain diameter, measuring system, mathematical model, estimation, feedback particle filter, online measurement, sensor

The trailing suction hopper dredger (TSHD) is a ship that excavates sediments from the sea bottom while sailing. Soil properties have a strong effect on the dredging process. The parameter with the greatest importance is the real-time soil grain diameter $d_{m}$. This, however, cannot be directly measured by available sensors. In this paper, an alternative method is proposed to solve this problem. A new estimation method is developed on the basis of an existing sedimentation model and measuring system data. The loading process with several grain diameters is simulated to perform a sensitivity analysis of soil type. Simulation results show that the dredging efficiency is strongly affected by fine soil. This soil-related estimation problem is solved with a continuous-discrete feedback particle filter (CD-FPF), which is a recently developed filter for a $\mathrm{CD}$ time system. For comparison, a bootstrap particle filter (BPF) is also used to simulate the steplike changes in $d_{m}$ in both the no-overflow and constantvolume loading phases. The results show that the CD-FPF outperforms the BPF in terms of accuracy and applicability. Thus, it is recommended to be applied in the estimator of artificial intelligence (AI) dredging systems.

\section{Introduction}

Recent research has shown that smart dredging is an effective approach that can lead to significant improvements in production optimization, pollution reduction, and labor saving for dredging processes. The integrated dredging control system (DCS), developed by the Royal IHC company, enables the efficient control of the vital dredging process through the use of adaptive self-learning methods. The artificial intelligence (AI) dredging system, ${ }^{(1)}$ which is the core structure of the DCS, contains a few estimators and a model predictive controller (MPC) to provide an optimal control strategy for a given situation.

*Corresponding author: e-mail: jqfu@staff.shu.edu.cn https://doi.org/10.18494/SAM.2019.2220 
The design of the MPC is based on several subsystems such as drag-head excavation, slurry transport, and hopper sedimentation. In general, the behavior of these systems is strongly determined by the soil type. ${ }^{(2-4)}$ These systems predict the dredging production by assuming that the soil characteristics are known. As the soil type changes continuously throughout the dredging cycle, it is often impossible to obtain the parameters associated with soil properties online by measurements. Instead, a few sophisticated algorithms can be selected to estimate these immeasurable parameters. ${ }^{(5)}$ The soil properties are estimated by measuring system data. The measuring system of the hopper dredge consists of pipeline density, pipeline flow, overflow tube height, draught, and liquid level sensors, and so on (see Figs. 1 and 2).

Most of the dredging-related parameters can be obtained from on-line measurements with these sensors. Unfortunately, some parameters cannot be obtained by direct measurement. Moreover, they play a vital role in dredging operations. Therefore, these parameters can only be estimated using algorithms, models, and data.

One typical application of estimation techniques is the overflow loss estimator, ${ }^{(1,5)}$ which is an AI-based module that is capable of estimating the overflow mixture density $\rho_{o}$ and flow rate $Q_{o}$. The estimation algorithms that are often used include the unscented Kalman filter (UKF) and bootstrap particle filter (BPF). It was found that the cascaded observer with the UKF/BPF for $Q_{o}$ and the BPF for $\rho_{o}$ is the best combination and had been implemented in the draught and loading monitor. ${ }^{(6,7)}$ In the case of the drag-head excavation process, more algorithms, such as the extended Kalman filter (KF), central difference filter, and Gaussian sum filter, were investigated to solve the problems of cutting estimation and the estimation of cutting and jetting. ${ }^{\left({ }^{(8)}\right.}$

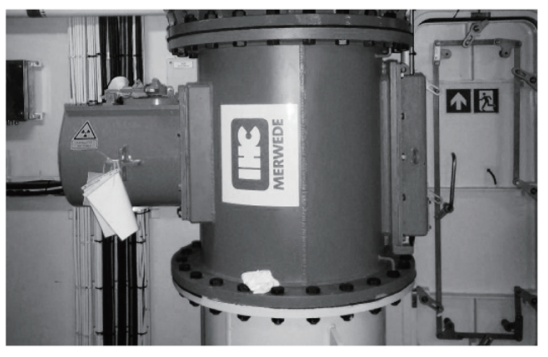

(a)

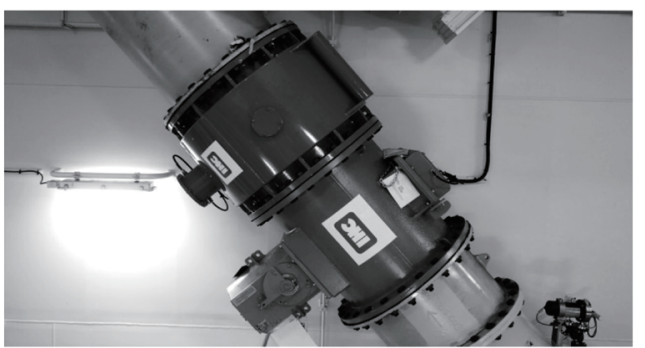

(b)

Fig. 1. (a) Pipeline density and (b) pipeline flow sensors.

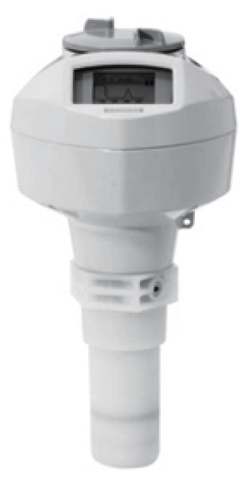

(a)

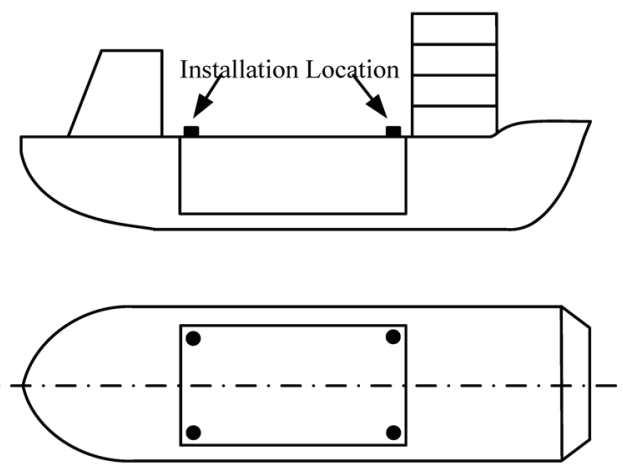

(b)

Fig. 2. Hopper mixture level sensor: (a) physical map and (b) installation location of the sensor. 
In our previous work, we showed that the pattern search and genetic algorithm can be used to solve the hopper estimation problem. Although these algorithms only provide offline estimates of soil parameters, the results have shown that both methods are effective and useful for different working conditions.

Recently, a real-time soil grain diameter estimator has been developed. ${ }^{(8)}$ Solutions to the estimation problem are obtained by dividing the overall loading process into three separate phases. The observer of the BPF was designed using the measured values of $Q_{o}$ and $\rho_{o}$ for the no-overflow and constant-volume phases. ${ }^{(9)}$ Moreover, the feedback particle filter (FPF) and BPF used for estimating immeasurable parameters for the no-overflow phase were compared. ${ }^{(8,10)}$ The results suggested that the FPF could become a benchmark filter for onboard estimators.

The continuous-FPF is based on mean-field optimal control techniques. ${ }^{(11-13)}$ It applies a feedback gain structure and avoids the resampling step, which is its main distinction from the conventional BPF. Note that the sampling time of the FPF is generally less than $0.05 \mathrm{~s}$ to retain the accuracy of outputs. ${ }^{(14)}$ However, the sampling period of the sensors onboard ranges from 1 to $30 \mathrm{~s}$. Thus, the limitation of the continuous-FPF in terms of sampling rate makes it unfeasible for the engineering practice of the AI system.

Motivated by the facts that have been presented above, in this paper, we focus on the recently developed continuous-discrete FPF (CD-FPF), ${ }^{(15)}$ the continuous-discrete version of FPF, to provide a solution to soil-related estimation problems for the first two loading phases. The rest of this paper is organized as follows. In Sect. 2, a framework for the online estimation of the soil grain diameter $d_{m}$ is described. The sensitivity analysis of the effect of the soil grain diameter $d_{m}$ on loading performance appears in Sect. 3. In Sect. 4, we introduce the CDFPF applied to the CD time nonlinear system. In Sect. 5, we present the results of numerical simulations and performance comparisons between the CD-FPF and the BPF. We conclude this paper in Sect. 6.

\section{Estimation Framework}

We start by introducing the online estimator based on the sedimentation model to predict the immeasurable parameters online including the soil grain diameter $d_{m}$, sand bed height $h_{s}$, and overflow density $\rho_{o}$.

The input signals are the incoming flow rate $Q_{i}$, the density of the incoming mixture, $\rho_{i}$, and the overflow weir height $h_{o}$, given by the measuring system (see Fig. 3). The initial soil grain diameter $d_{m, 0}$, indicating the average soil characteristic of the dredging area, can be calculated offline. The so-called prior predictions of the total mass $\hat{m}_{t}$ and height $\hat{h}_{t}$ are calculated with the state-space equations of the sedimentation model. Once the measurements of $m_{t}$ and $h_{t}$ are given, $d_{m}$ is calibrated and other immeasurable parameters can be estimated online.

\subsection{Sedimentation model}

Existing complex sedimentation models provide detailed descriptions of the physical phenomenon occurring in the hopper. ${ }^{(4,16)}$ These models are based on a large number of partial differential equations and uncertain environmental parameters, and thus cannot be used in the 


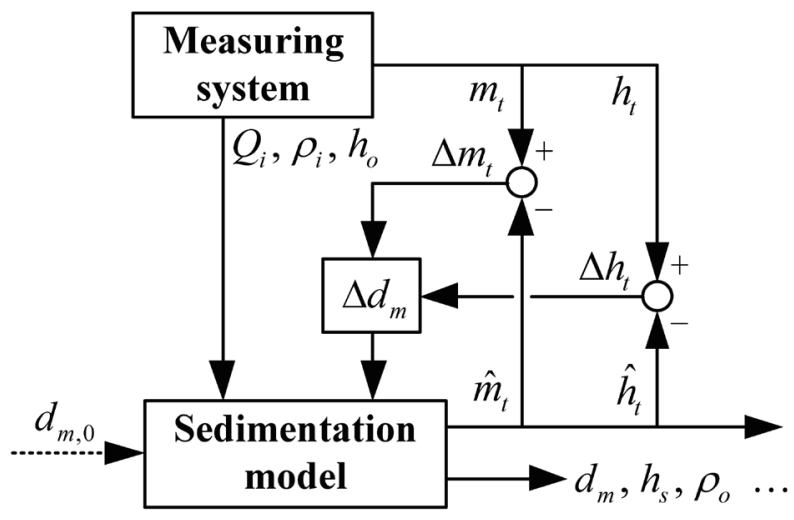

Fig. 3. Process of estimation based on sedimentation model.

AI dredging system. Consequently, we introduce an alternative model, the simplified onedimensional model in the vertical direction (1DV), ${ }^{(17)}$ to describe the sedimentation process.

The sedimentation process can be modeled using the following balance equations:

$$
\begin{aligned}
& \dot{m}_{t}=Q_{i} \rho_{i}-Q_{o} \rho_{o}, \\
& \dot{h}_{t}=\left(Q_{i}-Q_{o}\right) / S\left(h_{t}\right), \\
& \dot{h}_{s}=Q_{s} / S\left(h_{s}\right),
\end{aligned}
$$

where $Q_{s}$ denotes the settling sand flow rate in the hopper. The horizontal area $S$ depends on the size of the hopper and is related to the vertical height $h$ [i.e., $S=S(h)$ ].

Basically, there are two main methods for loading the hopper, namely, the constant volume system (CVS) and constant tonnage system (CTS). ${ }^{(18)}$ The CTS has an adjustable overflow weir that is lowered to keep the tonnage of the mixture constant if the content of the hopper reaches the maximum tonnage designed. For simplification, we focus on the CVS in this paper, which means that the overflow weir is fixed to make the effective volume of the mixture constant.

The settling sand flow rate $Q_{s}$, the main factor that affects the sand bed height, is associated with the erosion coefficient $k_{e}$, the terminal settling velocity of particles, $v_{s 0}$, and the hindered settling power $\beta$ as follows. ${ }^{(8,17)}$

$$
Q_{s}=\left[1-\left(\frac{Q_{o}}{k_{e}\left(h_{t}-h_{s}\right)}\right)^{2}\right] v_{s 0} \frac{V_{t}-V_{s}}{h_{t}-h_{s}} \frac{\rho_{m}-\rho_{w}}{\rho_{s}-\rho_{m}}\left(\frac{\rho_{q}-\rho_{m}}{\rho_{q}-\rho_{w}}\right)^{\beta},
$$

where $\rho_{w}\left(1.024\right.$ ton $\left./ \mathrm{m}^{3}\right)$ and $\rho_{q}\left(2.65 \mathrm{ton} / \mathrm{m}^{3}\right)$ denote the water and quartz densities, respectively. The sand bed density $\rho_{s}$ depends on the in situ soil type. The average density of the mixture above the sand bed, $\rho_{m}$, can be calculated as

$$
\rho_{m}=\frac{m_{t}-m_{s}}{V_{t}-V_{s}},
$$


where $V_{t}$ denotes the loading volume. $V_{s}$ and $m_{s}$ respectively correspond to the volume and mass of the sand bed, which settles at the bottom of the hopper.

Models for the prediction of $\rho_{o}$ have been proposed in Ref. 17. Experiments show that the water-layer model is the most suitable one in terms of practical use. The water-layer model assumes that a thin water layer is formed on top of the mixture soup layer, ${ }^{(17)}$ formed by upward water flow caused by the settling of grains. The displacement of a volume of settling grains invokes the same volume of water to flow in the opposite direction, i.e., the upward water flow rate $Q_{w}$ is equal to the solid flow rate $Q_{\text {solid }}$ :

$$
Q_{w}=Q_{\text {solid }}=\frac{\rho_{s}-\rho_{m}}{\rho_{q}-\rho_{w}} Q_{s} .
$$

The overflow rate $Q_{o}$ is the sum of $Q_{w}$ and the mixture soup flow rate above the sand bed, $Q_{m s}$; thus, the overflow density $\rho_{o}$ is given by

$$
\rho_{o}=\frac{\rho_{m} Q_{m s}+\rho_{w} Q_{w}}{Q_{m s}+Q_{w}}=\rho_{m}-\frac{Q_{w}}{Q_{o}}\left(\rho_{m}-\rho_{w}\right)
$$

\subsection{Soil-type-dependent parameters}

Uncertain soil parameters $\left(k_{e}, v_{s 0}\right.$, and $\left.\beta\right)$ that appear in Eq. (2) depend on the properties of excavated soil. Since it is too difficult to estimate these parameters simultaneously, we need to approximate them as functions of the soil grain diameter $d_{m}$. Some functions can be found in Ref. 17.

$$
\begin{aligned}
& v_{s 0}= \begin{cases}424 R_{s d} d_{m}^{2} & d_{m} \in(0,0.1] \mathrm{mm} \\
8.925\left(\sqrt{1+95 R_{s d} d_{m}^{3}}-1\right) / d_{m} & d_{m} \in(0.1,1] \mathrm{mm} \\
87 \sqrt{R_{s d} d_{m}} & d_{m} \in(1,12.8] \mathrm{mm}\end{cases} \\
& \beta=\frac{4.7+0.41 R e_{p}^{0.75}}{1+0.175 \operatorname{Re}_{p}^{0.75}}
\end{aligned}
$$

Here, the relative submerged density $R_{s d}$ and Reynolds number $R e_{p}$ are given by

$$
\begin{aligned}
& R_{s d}=\left(\rho_{q}-\rho_{w}\right) / \rho_{w}, \\
& R e_{p}=-2.289+41.53 d_{m}+118.6 d_{m}^{2} .
\end{aligned}
$$

The erosion coefficient $k_{e}$ is ${ }^{(12)}$

$$
k_{e}=W_{s h} \sqrt{8(1-n) \mu g R_{s d} d_{m} / f},
$$


where $W_{s h}$ is the width of the hopper, $n$ denotes the sand bed porosity, and $\mu$ is the internal friction of the sediment. $f$ is the friction factor and $g$ corresponds to the gravitational acceleration. In general, the accurate calculation of these parameters is not a straightforward task. Fortunately, $k_{e}$ can be calculated using the empirical formula as ${ }^{(8,9)}$

$$
k_{e}=28.06 \sqrt{d_{m}}-6.35
$$

\subsection{Estimation problem}

Owing to the time-varying characteristic and high uncertainty of $d_{m}$, no mathematical model exists currently to describe the real changes in $d_{m}$. As a consequence, we model it as a random walk, which is commonly applied in the stochastic problem ${ }^{(8-10)}$

$$
\dot{d}_{m}=w_{d m} .
$$

Here, $w_{d m}$ is a Wiener process with the standard deviation $\sigma_{d m}$.

In this paper, we assume that the sand bed height $h_{s}$ can be measured using the sensors under laboratory conditions. The values of other parameters, such as $Q_{i}, \rho_{i}, m_{t}$, and $h_{t}$, are available through the monitoring system. Considering that the overflow weir height $h_{o}$ is fixed, input and output vectors are defined as

$$
\boldsymbol{x}=\left[\begin{array}{lllllll}
m_{s} & h_{s} & V_{s} & h_{t} & m_{t} & V_{t} & d_{m}
\end{array}\right]^{\mathrm{T}}, \quad \boldsymbol{u}=\left[\begin{array}{lll}
Q_{i} & \rho_{i} & h_{o}
\end{array}\right]^{\mathrm{T}}, \quad \boldsymbol{z}=\left[\begin{array}{lll}
m_{t} & h_{t} & h_{s}
\end{array}\right]^{\mathrm{T}} .
$$

The state-space model is given by

$$
\begin{aligned}
& \mathrm{d} x_{1, t}= Q_{s, t} \rho_{s, t} \mathrm{~d} t+\mathrm{d} w_{1, t}, \\
& \mathrm{~d} x_{2, t}= Q_{s, t} / S\left(x_{2, t}\right) \mathrm{d} t+\mathrm{d} w_{2, t}, \\
& \mathrm{~d} x_{3, t}= Q_{s, t} \mathrm{~d} t+\mathrm{d} w_{3, t}, \\
& \mathrm{~d} x_{4, t}=\left(u_{1, t}-Q_{o, t}\right) / S\left(x_{4, t}\right) \mathrm{d} t+\mathrm{d} w_{4, t}, \\
& \mathrm{~d} x_{5, t}=\left(u_{1, t} u_{2, t}-Q_{o, t} \rho_{o, t}\right) \mathrm{d} t+\mathrm{d} w_{5, t}, \\
& \mathrm{~d} x_{6, t}=\left(u_{1, t}-Q_{o, t}\right) \mathrm{d} t+\mathrm{d} w_{6, t}, \\
& \mathrm{~d} x_{7, t}=0 \mathrm{~d} t+\mathrm{d} w_{7, t} \\
& z_{1, t}=x_{5, t}+v_{1, t}, \\
& z_{2, t}=x_{4, t}+v_{2, t}, \\
& z_{3, t}=x_{2, t}+v_{3, t},
\end{aligned}
$$

where $w_{t}$ and $v_{t}$ correspond to the process noise and measurement noise, respectively. The settling sand flow rate $Q_{s}$ and overflow density $\rho_{o}$ are calculated through the $1 \mathrm{DV}$ and waterlayer models, respectively. 


\section{Sensitivity of Soil Type}

In this section, we determine which soil type mainly affects the dredging efficiency.

\subsection{Dredging indices}

We choose the following four indices to quantify the dredging performance: the sand bed height $h_{s}$, the overflow density $\rho_{o}$, the weight of dry solids, TDS, and the sand storage ratio SSR.

$$
\begin{aligned}
T D S_{t} & =\frac{\rho_{q}}{\rho_{q}-\rho_{w}} \int_{0}^{t}\left[Q_{i, \tau}\left(\rho_{i, \tau}-\rho_{w}\right)-Q_{o, \tau}\left(\rho_{o, \tau}-\rho_{w}\right)\right] d \tau, \\
S S R_{t} & =1-\frac{\int_{0}^{t} Q_{o, \tau}\left(\rho_{o, \tau}-\rho_{w}\right) d \tau}{\int_{0}^{t} Q_{i, \tau}\left(\rho_{i, \tau}-\rho_{w}\right) d \tau} .
\end{aligned}
$$

The calculations of $h_{s}$ and $\rho_{o}$ have been presented in Sect. 2. TDS is a standard index frequently used in the dredging industry. SSR ranges from 0 to $100 \%$. A low SSR means huge overflow losses, i.e., poor dredging performance. In contrast, an SSR of 100\% indicates that all the incoming materials remain in the hopper.

\subsection{Parameters of loading simulations}

The hopper, used for simulating the loading process, is assumed to be a rectangular parallelepiped, approximated with the principal dimensions of the dredger Xin Hai Feng. For the CVS loading method, $Q_{o}$ is equal to its value in the constant-volume phase (i.e., $Q_{o}=Q_{i}$ while overflowing). To obtain the true state of simulation, the noise in the process and observation is considered to be zero. For simplification, $Q_{i}$ and $\rho_{i}$ are assumed to be constant. The initial height of water in the hopper, $h_{t, 0}$, is $4.7 \mathrm{~m}$. The sand bed density $\rho_{s}$ is set to $1.95 \mathrm{ton} / \mathrm{m}^{3}$. The total simulation time $T$ is set to $90 \mathrm{~min}$. Table 1 shows the vital parameters of simulations.

For carrying out the sensitivity analysis, we investigate several soil types with grain sizes ranging from 0.6 to $1.00 \mathrm{~mm}$ (Table 2).

Table 1

Vital parameters of loading simulations.

\begin{tabular}{ccccccc}
\hline$S\left(\mathrm{~m}^{2}\right)$ & $h_{o}(\mathrm{~m})$ & $h_{t, 0}(\mathrm{~m})$ & $Q_{i}\left(\mathrm{~m}^{3} / \mathrm{s}\right)$ & $\rho_{i}\left(\right.$ ton $\left./ \mathrm{m}^{3}\right)$ & $\rho_{s}\left(\right.$ ton $\left./ \mathrm{m}^{3}\right)$ & $T(\mathrm{~min})$ \\
\hline 1080 & 15.45 & 4.7 & 12 & 1.35 & 1.95 & 90 \\
\hline
\end{tabular}

Table 2

Soil grain diameters for simulations.

\begin{tabular}{lc}
\hline Soil type & $d_{m}(\mathrm{~mm})$ \\
\hline Fine & $0.06-0.20$ \\
Medium & $0.20-0.60$ \\
Coarse & $0.60-1.00$ \\
\hline
\end{tabular}




\subsection{Sensitivity analysis}

Sensitivity analysis is performed to study the effect of soil type on dredging performance. From Fig. 4, it can be seen that the fine soil with low settling efficiency forms a thin layer at the bottom of the hopper, resulting in a high initial overflow density. The effect of scour is clearly visible and $\rho_{o}$ increases in the constant-volume phase. The coarse soil with high settling efficiency leads to a lower mixture density above the sand bed, which means that the initial overflow density of this soil type is close to that of water. In practice, the loading time is associated with the soil type: the finer the sand, the longer the loading time. Again, TDS means the weight of the solids, excluding the pore water and the water on top of the sand bed. It can be seen that TDS is weakly associated with the soil type in the no-overflow phase, while it is mainly determined by the overflow density during the constant-volume process. Similar correlations with soil type and overflow density are also observed in the case of SSR. When $\rho_{o}$ reaches $\rho_{i}$, the overflow losses become so huge that it is no longer economically feasible to continue dredging.

To summarize, the cumulative overflow losses are weakly related to coarse soil. Thus, the dredging optimization ought to focus on the process of drag-head excavation and slurry

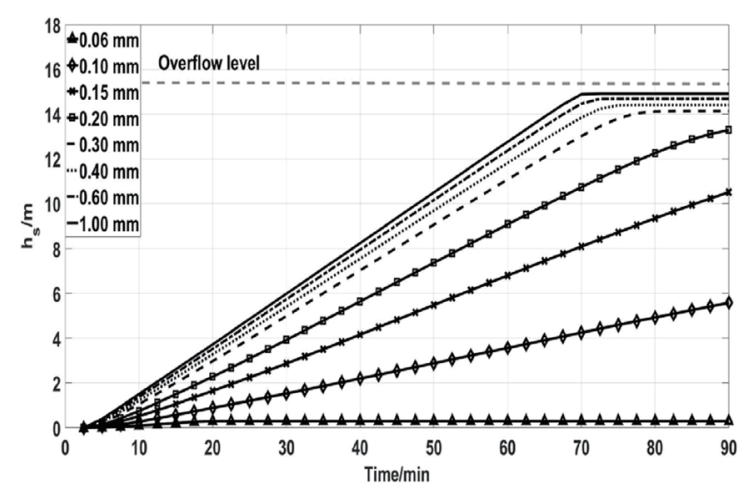

(a)

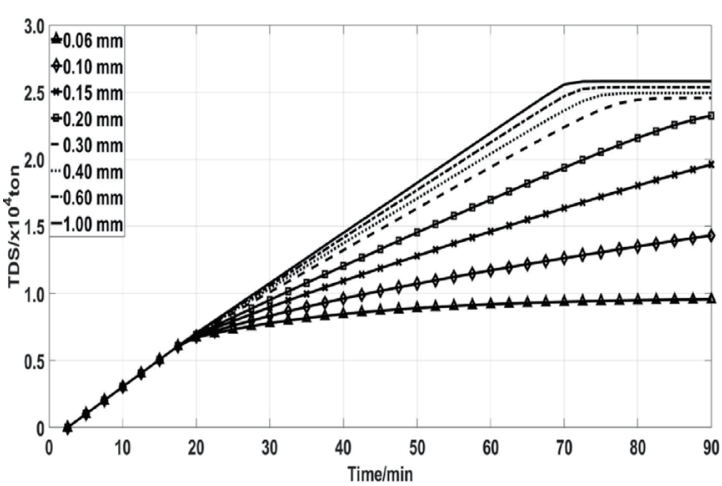

(c)

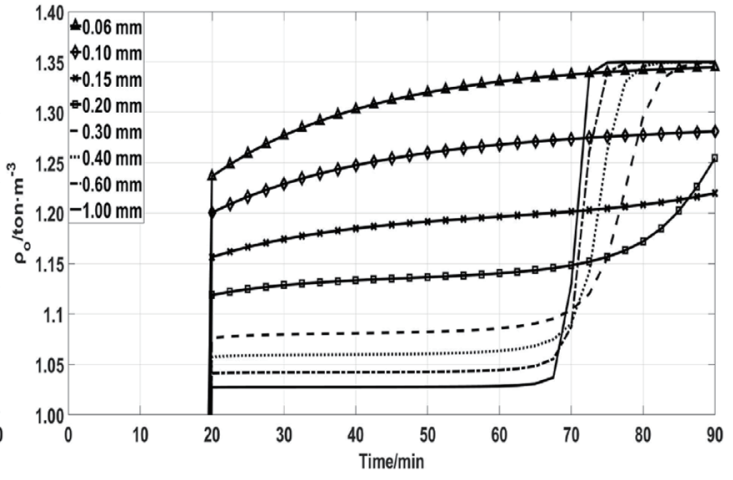

(b)

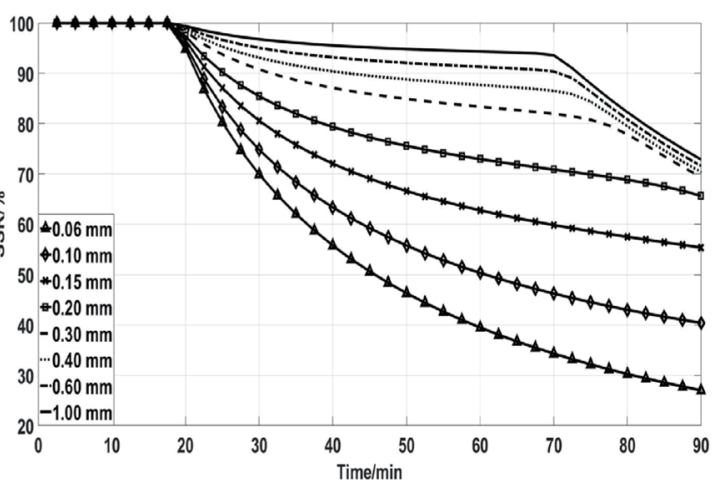

(d)

Fig. 4. Results of dredging evaluations with several $d_{m}$ values: (a) sand bed height, (b) overflow density, (c) TDS, and (d) sand storage ratio. 
transport. For fine/medium soil, the overflow losses are huge and the optimization of the sedimentation process is indispensable. The particle size distribution of this soil type has a considerable effect on the dredging operation. Therefore, it is extremely important to estimate the soil grain diameter in this case.

\section{Estimation Method}

In this section, we introduce the CD-FPF to be applied in engineering practice.

Suppose that the CD time system is

$$
\begin{aligned}
& d \boldsymbol{x}_{t}=\boldsymbol{f}_{t}\left(\boldsymbol{x}_{t}, \boldsymbol{u}_{t}\right) d t+d \boldsymbol{\beta}_{t}, \\
& \boldsymbol{z}_{n}=\boldsymbol{h}\left(\boldsymbol{x}_{t_{n}}\right)+\boldsymbol{e}_{n},
\end{aligned}
$$

where $x_{t} \in R^{d}$ is the state at the time $t$, which evolves in the form of continuous time, $z_{n} \in R^{m}$ is the discrete-time measurement obtained at the time $t_{n}$, and $\boldsymbol{u}_{\boldsymbol{t}}$ is the input signal. Moreover, $\boldsymbol{f}$ and $\boldsymbol{h}$ are the state and measurement functions determined using the specified model, and $\boldsymbol{\beta}$ and $\boldsymbol{e}$ are the process noise and measurement noise, respectively. Both the process noise and the measurement noise are assumed to exhibit Gaussian distributions with the zero mean and covariance matrices $\boldsymbol{Q}$ and $\boldsymbol{R}$. The objective of filtering is to obtain the posterior distribution of $x_{t}$ given the observation history $\mathcal{L}_{t}:=\sigma\left\{z_{n}: \forall n, t_{n} \in[0, t]\right\}$.

Distinguishing from the continuous-FPF, the CD-FPF is a controlled system that transforms the discrete-time Bayesian measurement update to a continuously evolving process. It works in two steps: for $n=1,2,3, \ldots$,

Step 1: Continuous-time evolution of the state equation for $t \in\left[t_{n-1}, t_{n}\right)$;

Step 2: Discrete-time Bayesian update at the discrete time $t=t_{n}$.

For $t \in\left[t_{n-1}, t_{n}\right)$, the evolution of the $i$-th particle is described as

$$
d \boldsymbol{x}_{t}^{i}=\boldsymbol{f}_{t}\left(\boldsymbol{x}_{t}^{i}, \boldsymbol{u}_{t}\right) d t+d \boldsymbol{\beta}_{t}^{i},
$$

for $i=1, \ldots, N$, where $N$ is the number of particles. The initial state $\boldsymbol{x}_{t_{n-1}}^{i}$ is assumed to be known and $\boldsymbol{x}_{t_{n}^{-}}^{i}$ is defined as the right limit of $\boldsymbol{x}_{t}^{i}: \boldsymbol{x}_{t_{n}^{-}}^{i}=\lim _{t \rightarrow t_{n}} \boldsymbol{x}_{t^{i}}^{i}$.

At the discrete time $t_{n}$ when the $n$-th measurement $z_{n}$ is given, a particle flow $\boldsymbol{S}_{n}^{i}(\lambda)$ is introduced as

$$
\frac{d \boldsymbol{S}_{n}^{i}(\lambda)}{d \lambda}=\boldsymbol{K}_{n}^{i}(\lambda) \boldsymbol{I}_{n}^{i}(\lambda)
$$

where $\lambda \in[0,1]$ is the pseudo-time variable. The initial condition is set as $\boldsymbol{S}_{n}^{i}(0)=\boldsymbol{x}_{t_{n}^{-}}^{i}$, and the initial state for the next time interval $\boldsymbol{x}_{t_{n}}^{i}$ is set as $\boldsymbol{x}_{t_{n}}^{i}=\boldsymbol{S}_{n}^{i}(1) . \boldsymbol{K}_{n}^{i}(\lambda)$ is the feedback gain 
function and the innovation error $\boldsymbol{I}_{n}^{i}(\lambda)$ is given by

$$
\boldsymbol{I}_{n}^{i}(\lambda)=\boldsymbol{z}_{n}-\frac{1}{2}\left[\boldsymbol{h}\left(\boldsymbol{S}_{n}^{i}(\lambda)\right)+\hat{\boldsymbol{h}}^{*}(\lambda)\right]
$$

where $\hat{\boldsymbol{h}}^{*}(\lambda):=(1 / N) \sum_{i=1}^{N} \boldsymbol{h}\left(\boldsymbol{S}_{n}^{i}(\lambda)\right)$.

It can be seen that Eq. (16) is similar to the innovation process that appears in the classical KF. Actually, the CD-FPF provides a generalization of the KF to a general class of continuousdiscrete nonlinear problems. The solution of the feedback gain function $\boldsymbol{K}_{n}^{i}(\lambda)$ is the central problem of the CD-FPF algorithm. In practical use, $\boldsymbol{K}_{n}^{i}(\lambda)$ is generally approximated with numerical algorithms instead of being computed explicitly. In this paper, the constant gain approximation is chosen for the solution to $\boldsymbol{K}_{n}^{i}(\lambda){ }^{(13)}$ which means that each particle has the same feedback gain function

$$
\boldsymbol{K}_{n}(\lambda)=\left[\boldsymbol{K}_{d \times m}\right]_{n}^{i}(\lambda)=\frac{1}{N}\left(\sum_{j=1}^{N} \boldsymbol{S}_{n, p}^{j}(\lambda)\left[\boldsymbol{h}\left(\boldsymbol{S}_{n, q}^{j}(\lambda)\right)-\hat{\boldsymbol{h}}_{q}(\lambda)\right]\right) \boldsymbol{R}^{-1}
$$

for $p=1,2, \ldots, d$ and $q=1,2, \ldots, m$. Recall that $d$ and $m$ are the dimensions of the state and observation vector, respectively, and the constant gain function $\boldsymbol{K}_{n}(\lambda)$ is a $d \times m$ matrix.

To simulate the CD-FPF, we use the Euler discretization scheme for both the real time step $\Delta t$ and the pseudo time step $\Delta \lambda$. Note that the gain function should be approximated at each step of $\lambda$ according to Eq. (17). In fact, the algorithm for $\lambda$ is conceptually the same as that described for the $t$ of the continuous-FPF. ${ }^{(15)}$ The general CD-FPF is presented in Algorithm 1.

\section{Numerical Simulations}

In this section, we provide the estimates of the soil $d_{m}$ obtained with the CD-FPF. For comparison, we consider the conventional BPF and compare the performance characteristics of the two filters. More information about the BPF can be found in Ref. 19 and details are omitted here to save space.

\subsection{Setting}

To simulate the dredging operation, the sampling period $\Delta t_{n}$ of filters ranges from 1 to 10 s. The real-time step $\Delta t$ and pseudo time step $\Delta \lambda$ of the CD-FPF are both set to $0.05 \mathrm{~s}$. For the CVS loading simulation, true states are generated without the process noise. The BPF applies the systematic resampling algorithm. For the application of filters onboard, the cost of the computation of filters should be considered. Therefore, for the BPF, we use 100 particles, while we use 20 particles in the experiments associated with the CD-FPF.

In general, the measurement noise is mainly determined by the accuracy of the sensor. On the other hand, the process noise in system (11a) is crucial for filters to have a chance of 


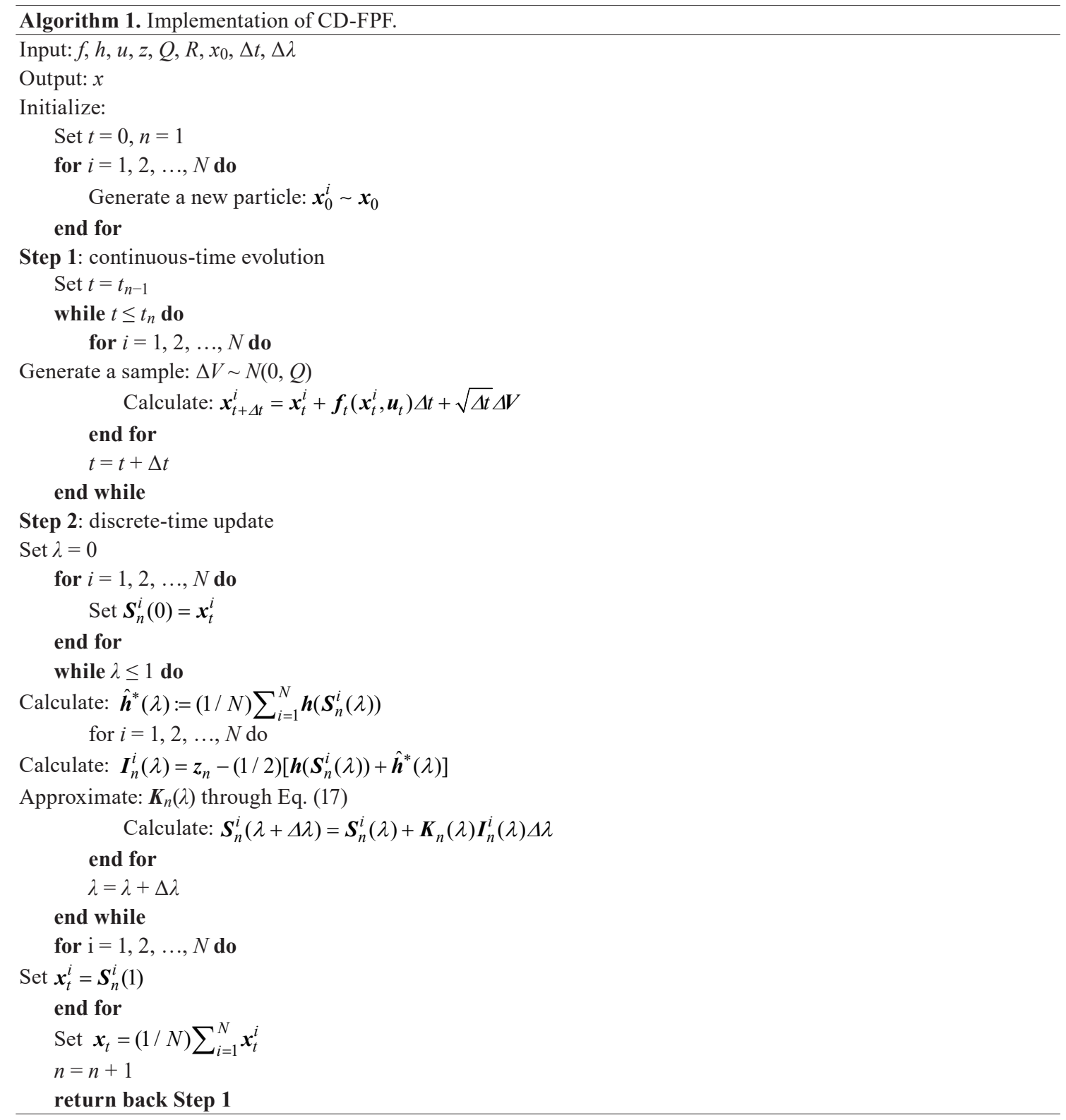

estimating $d_{m}$. Recall that coarse soil is very weakly sensitive to the dredging performance; hence, we focus on fine/medium soil where the grain diameter is in the range of $d_{m} \in[0.1,0.3]$ $\mathrm{mm}$. The process noise of the grain diameter, $w_{7}$, is selected in several cases to determine the sensitivity of the filters to it. Other parameters of the filters are presented in Table 3.

Owing to the switching nature of the loading process, we split the sedimentation problem into two phases. In each phase, we consider a scenario where the dredger crosses over an area where the soil type changes rapidly, with the change in $d_{m}$ set to be steplike. To investigate the robustness of both filters with respect to initial conditions, the initial grain diameters of the filters are set with an offset of $+0.10 \mathrm{~mm}$ in each case, which reflects that the off-line calculated grain diameter deviates from its real value. 
Table 3

Parameters of filters.

\begin{tabular}{|c|c|c|c|c|c|c|c|c|c|}
\hline Filter & $\begin{array}{l}\text { Number of } \\
\text { particles, } N\end{array}$ & $\begin{array}{l}\text { Resampling } \\
\text { algorithm }\end{array}$ & \multicolumn{4}{|c|}{ Process noise $\boldsymbol{w}$} & \multicolumn{3}{|c|}{ Observation noise $\boldsymbol{v}$} \\
\hline BPF & 100 & Systematic & $w_{1} /$ ton & $w_{2} / \mathrm{m}$ & $w_{3} / \mathrm{m}^{3}$ & $w_{7} / \mathrm{mm}$ & $v_{1} /$ ton & $v_{2} / \mathrm{m}$ & $v_{3} / \mathrm{m}$ \\
\hline CD-FPF & 20 & None & 10 & 0.005 & 5 & $0.005-0.020$ & 1 & 0.05 & 0.10 \\
\hline
\end{tabular}

\subsection{Results and discussion}

The performance metrics of the filters we use are the root-mean-square error (RMSE) of $d_{m}$ over the time $t_{n}$ and the time-averaged RMSE

$$
\begin{aligned}
& R M S E_{t n}=\sqrt{\frac{1}{N} \sum_{i=1}^{N}\left(\hat{x}_{7, t_{n}}^{i}-x_{7, t_{n}}\right)^{2}}, \\
& R M S E=\sqrt{\frac{1}{T} \int_{t_{n}=0}^{T}\left[\frac{1}{N} \sum_{i=1}^{N}\left(\hat{x}_{7, t_{n}}^{i}-x_{7, t_{n}}\right)^{2}\right] d t .}
\end{aligned}
$$

Here, $x_{7, t_{n}}$ denotes the true simulated state of $d_{m}$ and $\hat{x}_{7, t_{n}}^{i}$ corresponds to the estimate at the time $t_{n}$ for the $i$-th particle.

Through the simulation of steplike changes in the true state of the soil $d_{m}$, it is possible to compare the performance characteristics of the filters for both loading processes. Figures 5 and 6 show the estimates with the process noise $w_{7}=0.005$ and $0.010 \mathrm{~mm}$, where $\Delta t_{n}=1 \mathrm{~s}$. Figure 7 depicts the results obtained with the sampling time $\Delta t_{n}=2 \mathrm{~s}$, where $w_{7}=0.005 \mathrm{~mm}$.

The $\mathrm{RMSE}_{t n}$ values of the two filters with the process noise $w_{7}=0.005$ and $0.010 \mathrm{~mm}$ are presented in Figs. 8 and 9, respectively. For the constant-volume phase, both the BPF and the CD-FPF perform similarly and produce highly accurate estimates. For the no-overflow phase, the RMSE tn $_{n}$ values of the CD-FPF are significantly smaller than those of the BPF, which suggests that the estimates obtained with the CD-FPF are more accurate than those obtained with the BPF. At the beginning of the loading, the CD-FPF converges faster to the true state than the BPF does. With the marked changes in true $d_{m}$, we can see that the estimates of the CD-FPF are faster in tracking the true state of the system. Table 4 shows a summary of the RMSEs of the filters with more values of $w_{7}$, where all sampling times are set to be $1 \mathrm{~s}$. The RMSEs of both filters are similar for the constant-volume phase. For the no-overflow phase, the estimates of the BPF are sensitive to $w_{7}$, while the accuracy of the estimates produced with the CD-FPF is nearly constant regardless of the choice of $w_{7}$.

From Figs. 6-10, one can observe that the estimate errors of the BPF are clearly associated with the sampling time $\Delta t_{n}$ for both loading phases. Table 5 gives the summary of RMSEs with sampling periods ranging from 1 to $10 \mathrm{~s}$, where $w_{7}=0.005 \mathrm{~mm}$. It can be seen that the higher the frequency of measurements, the higher the accuracy of estimates obtained with the filters. As the sampling time grows, the estimate error increases. If the sampling time is more than $2 \mathrm{~s}$, the estimates are relatively noisy and divergent for the BPF, while the RMSEs of the CD-FPF 


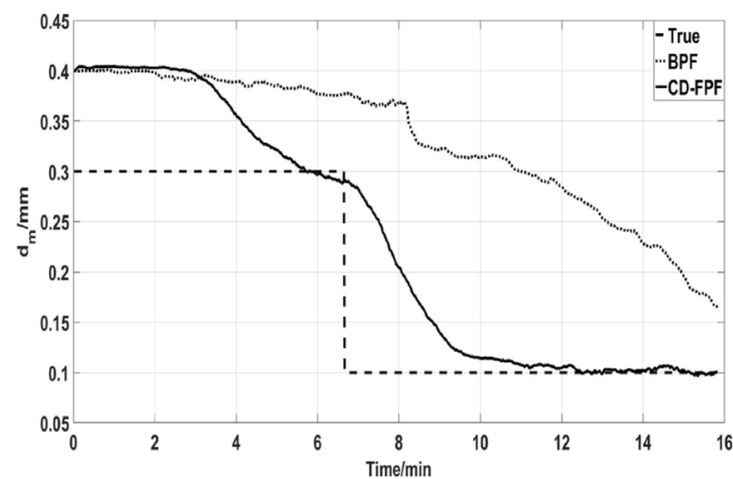

(a)

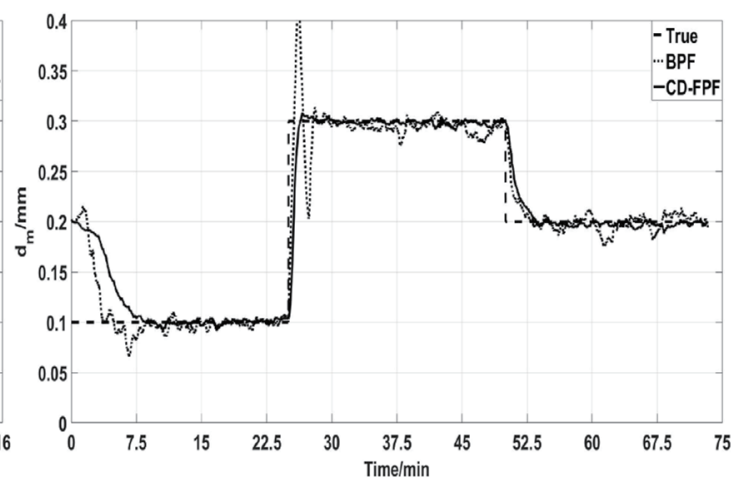

(b)

Fig. 5. Estimates of filters with $w_{7}=0.005 \mathrm{~mm}$ and $\Delta t_{n}=1 \mathrm{~s}$ : (a) no-overflow phase and (b) constant-volume phase.

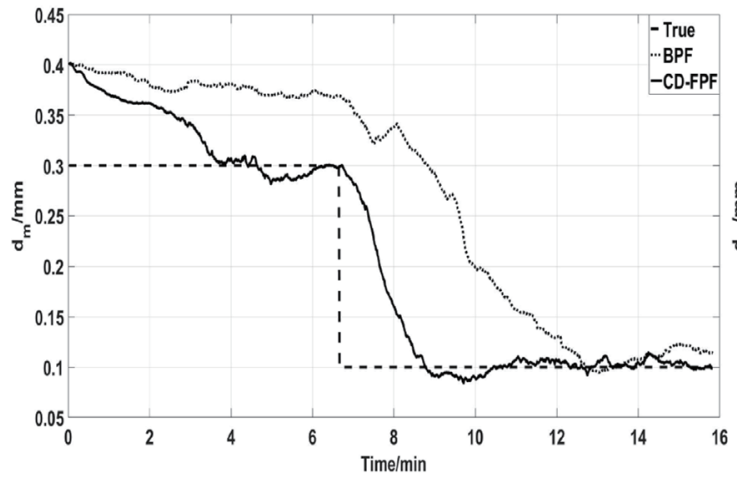

(a)

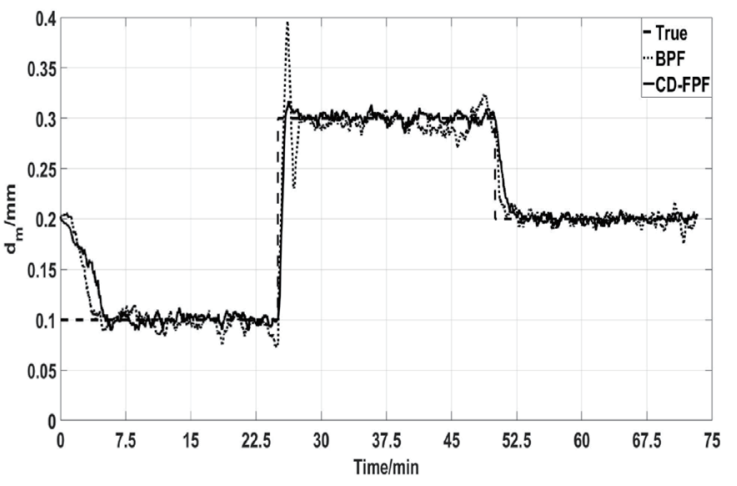

(b)

Fig. 6. Estimates of filters with $w_{7}=0.010 \mathrm{~mm}$ and $\Delta t_{n}=1 \mathrm{~s}$ : (a) no-overflow phase and (b) constant-volume phase.

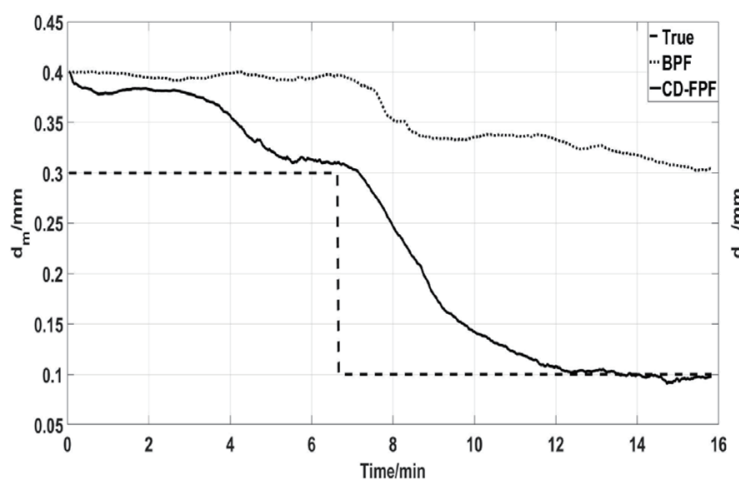

(a)

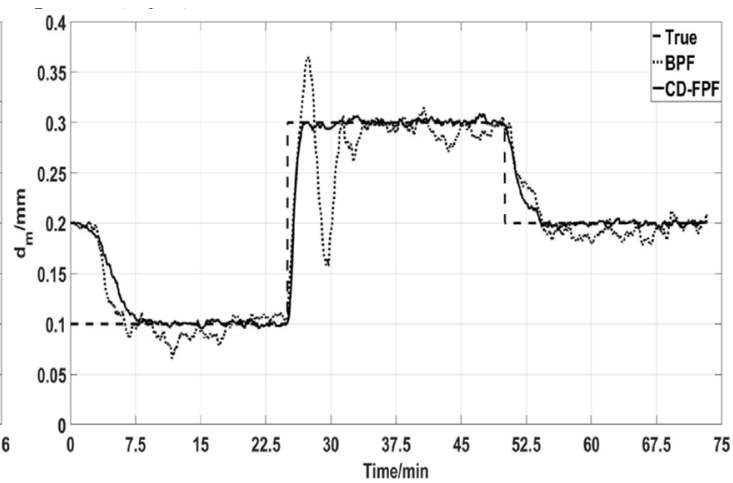

(b)

Fig. 7. Estimates of filters with $w_{7}=0.005 \mathrm{~mm}$ and $\Delta t_{n}=2 \mathrm{~s}$ : (a) no-overflow phase and (b) constant-volume phase. 


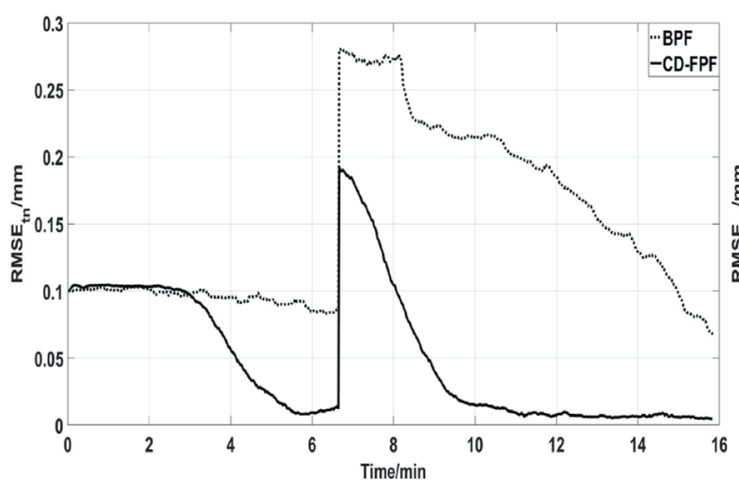

(a)

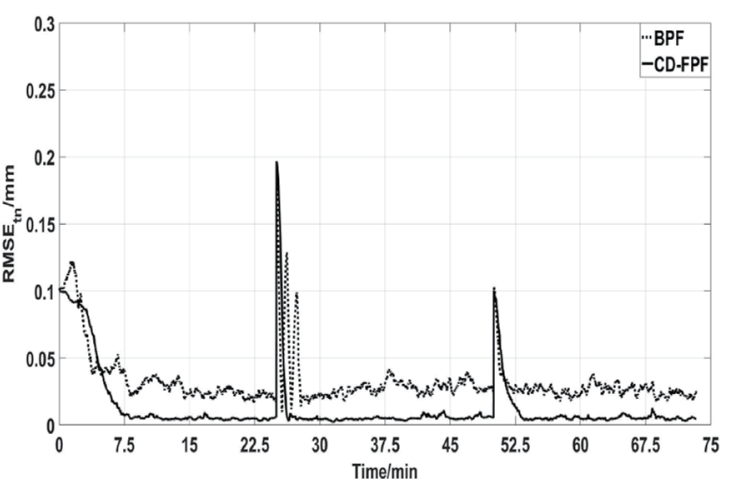

(b)

Fig. 8. RMSE $E_{t n}$ values of filters with $w_{7}=0.005 \mathrm{~mm}$ and $\Delta t_{n}=1 \mathrm{~s}$ : (a) no-overflow phase and (b) constant-volume phase.

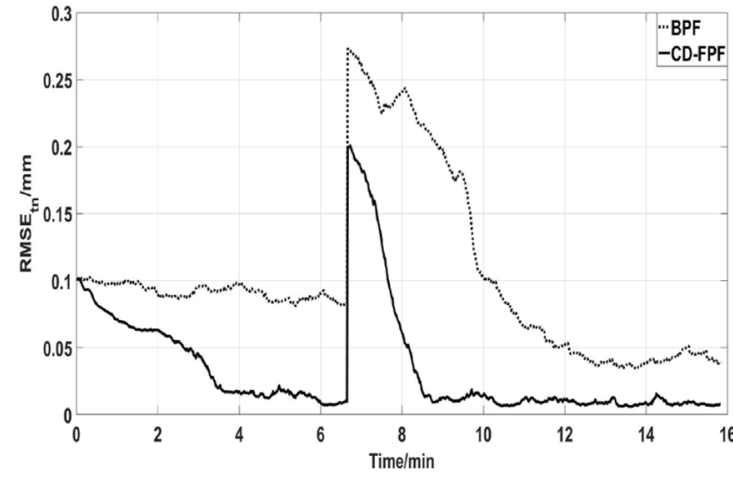

(a)

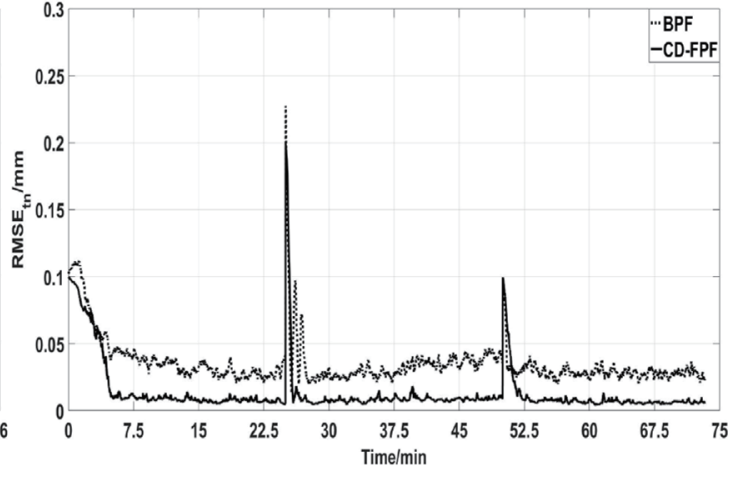

(b)

Fig. 9. RMSE $E_{t n}$ values of filters with $w_{7}=0.010 \mathrm{~mm}$ and $\Delta t_{n}=1 \mathrm{~s}$ : (a) no-overflow phase and (b) constant-volume phase.

Table 4

Summary of RMSE values with four choices of $w_{7}\left(\Delta t_{n}=1 \mathrm{~s}\right)$.

\begin{tabular}{|c|c|c|c|c|c|c|c|c|}
\hline \multirow{2}{*}{ Algorithm } & \multicolumn{4}{|c|}{ No-overflow phase } & \multicolumn{4}{|c|}{ Constant-volume phase } \\
\hline & $0.005 \mathrm{~mm}$ & $0.010 \mathrm{~mm}$ & $0.015 \mathrm{~mm}$ & $0.020 \mathrm{~mm}$ & $0.005 \mathrm{~mm}$ & $0.010 \mathrm{~mm}$ & $0.015 \mathrm{~mm}$ & $0.020 \mathrm{~mm}$ \\
\hline $\mathrm{PF}$ & 0.1592 & 0.0862 & 0.0514 & 0.0682 & 0.0171 & 0.0149 & 0.0151 & 0.0169 \\
\hline CD-FPF & 0.0475 & 0.0297 & 0.0205 & 0.0309 & 0.0115 & 0.0114 & 0.0101 & 0.0111 \\
\hline
\end{tabular}

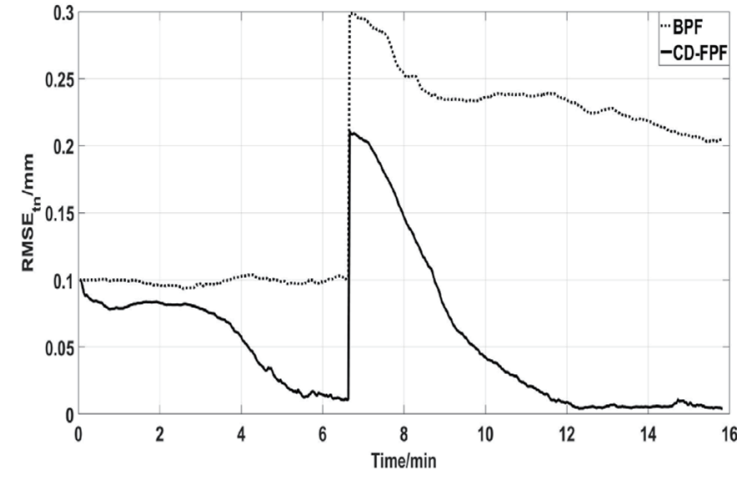

(a)

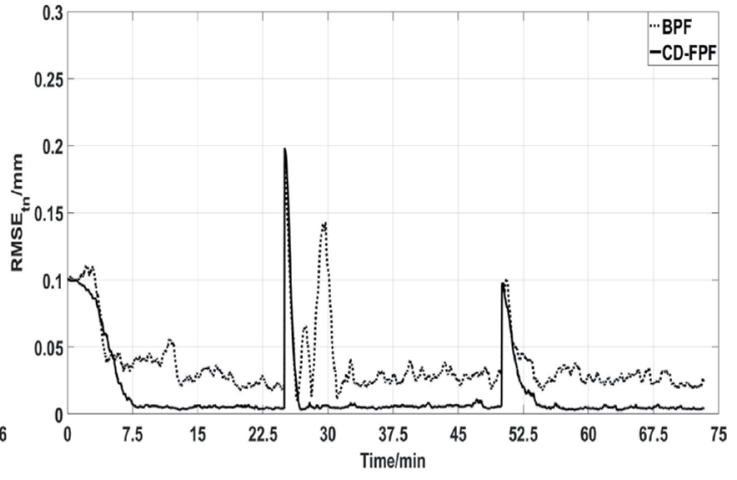

(b)

Fig. 10. $\mathrm{RMSE}_{t n}$ values of filters with $w_{7}=0.005 \mathrm{~mm}$ and $\Delta t_{n}=2 \mathrm{~s}$ : (a) no-overflow phase and (b) constant-volume phase. 
Table 5

Summary of RMSEs with four choices of $\Delta t_{n}\left(w_{7}=0.005 \mathrm{~mm}\right)$.

\begin{tabular}{|c|c|c|c|c|c|c|c|c|}
\hline \multirow{2}{*}{ Algorithm } & \multicolumn{4}{|c|}{ No-overflow phase } & \multicolumn{4}{|c|}{ Constant-volume phase } \\
\hline & $1 \mathrm{~s}$ & $2 \mathrm{~s}$ & $5 \mathrm{~s}$ & $10 \mathrm{~s}$ & $1 \mathrm{~s}$ & $2 \mathrm{~s}$ & $5 \mathrm{~s}$ & $10 \mathrm{~s}$ \\
\hline BPF & 0.1592 & 0.1808 & 0.2206 & 0.2209 & 0.0171 & 0.0181 & 0.0988 & 0.1100 \\
\hline CD-FPF & 0.0475 & 0.0638 & 0.0988 & 0.1115 & 0.0115 & 0.0143 & 0.0147 & 0.0161 \\
\hline
\end{tabular}

are nearly constant in the constant-volume phase. For the no-overflow phase, the estimates of the CD-FPF are within an acceptable range.

\section{Conclusion}

In this paper, we introduce an estimator of the soil grain diameter based on a 1DV sedimentation model and measuring system data. To determine which excavated soil mainly affects the dredging operation, we carry out the sensitivity analysis of soil type, which shows that the dredging performance is sensitive to medium/fine soil.

To overcome the estimation problem, we employ the CD-FPF used in a continuous-discrete nonlinear system. To compare the performance characteristics of the BPF and CD-FPF, we consider a steplike change in grain diameter, which models the marked changes in soil type in the dredging operation. Numerical simulations show that, in general, the estimates produced by the CD-FPF are more accurate than those obtained with the BPF, and that the CD-FPF converges faster to the true state than the BPF does. On the other hand, the choice of process noise $\mathrm{w}_{7}$ strongly affects the accuracy of the BPF, especially for the no-overflow phase. At a low signal sampling frequency, the estimates of the BPF diverge from the true state, while those of the CD-FPF maintain excellent estimation accuracy.

From simulation results, we conclude that the CP-FPF provides a satisfactory solution to the soil-related estimation problem and is suggested to be applied in an AI dredging system in future development.

Note that the loading method we used is the CVS; practically, modern dredgers have been equipped with a constant-tonnage controller to discharge a low-density mixture before the loading operation ends. Therefore, it is necessary to provide a solution to the estimation problem of the constant-tonnage loading process. Moreover, in this paper, we consider steplike changes in soil type during dredging in a real operation; the changes in soil type are smoother and the performance of the CD-FPF should be investigated in this case.

As mentioned previously, the performance of the CD-FPF is strongly determined by the selected feedback gain function. The improvement in estimation accuracy may result in degraded computational efficiency and the best approach to solving this problem will be studied in our future research.

\section{Acknowledgments}

This research was supported by a grant (No.17511107002) from the Science and Technology Commission of Shanghai Municipality of China and Natural Science Fund for Colleges and Universities in Jiangsu Province (No. 18KJB580005). 


\section{Author Contributions}

Z. Su analyzed the data and wrote most of the manuscript, including quality assurance and control; Z. X. Zhou contributed to writing the manuscript; M. H. Yu and W. Yuan helped to revise the manuscript; J.Q. Fu designed the study.

\section{Conflicts of Interest}

The authors declare no conflict of interest.

\section{References}

1 R. Mourik and J. Osnabrugge: Proc. WEDA XXXIV Technical Conf. TAMU 45 Dredging Seminar (2014).

2 P. C. Nie, T. Dong, Y. He, and S. P. Xiao: Sensors 18 (2018) 2. https://doi.org/ 10.3390/s18020391

3 X. Chen, S. Miedema, and C. Rhee: The 7th World Congr. Particle Technology (WCPT7) (2014) 804. https:// doi.org/10.1016/j.proeng.2015.01.194

4 C. Rhee: Dr. Thesis, Delft University of Technology (2002).

5 J. Braaksma, J. Osnabrugge, and C. Keizer: Ceda Dredging Days (Netherlands, 2009) 102-113. https://doi.org/ 10.1139/T08-107

6 R. Babuska, Z. Lendek, J. Braaksma, and C. D. Keizer: Proc. American Control Conf. (IEEE, 2006) 5751. https://doi.org/10.1109/acc.2006.1657642

7 Z. Lendek, R. Babuska, J. Braaksma, and C. D. Keizer: Control Engineering Practice (IEEE, 2008) 392. https://doi.org/10.1016/j.conengprac.2007.05.004

8 P. Stano: Dr. Thesis, Delft University of Technology (2013).

9 P. Stano, Z. Lendek, R. Babuska, and J. Braaksma: Proc. IEEE Conf. Control Applications (IEEE, 2010) 292. https://doi.org/10.1109/cca.2010.5611339

10 P. Stano, A. Tilton, and R. Babuska: Control Eng. Pract. 24 (2014) 67. https://doi.org/10.1016/ j.conengprac.2013.11.005

11 T. Yang, P. Mehta, and S. Meyn: 50th IEEE Conf. Decision and Control and European Control Conf. (CDC-ECC) (IEEE, 2011) 7909. https://doi.org/10.1109/cdc.2011.6160950

12 J. Dampf, K. Frankl, and T. Pany: Sensors 18 (2018) 2736. https://doi.org/10.3390/s18082736

13 T. Yang, P. Mehta, and S. Meyn: Trans. Autom. Control. 58 (2013) 2465. https://doi.org/10.1109/ tac.2013.2258825

14 H. X. Zhang, S. H. Zhou, and S. M. Feng: Acta Electronica Sinica 44 (2016) 95. https://doi.org/10.3969/ j.issn.0372-2112.2016.01.014

15 T. Yang, H. Blom, and P. Mehta: American Control Conf. 58 (2014) 648. https://doi.org/10.1109/ ACC.2014.6859259

16 Y. C. Hao, G. J. Hong, and C. Wang: Port Waterway Eng. 12 (2012) 119. http://doi:10.3969/ j.issn.1002-4972.2012.12.025

17 J. Braaksma: Dr. Thesis, Delft University of Technology (2008).

18 S. Miedema: OE4607 Introduction Dredging Engineering, Delft University of Technology (2015).

19 C. Zhang, A. Taghvaei, Mehta, and G. Prashant, IEEE Trans. Autom. Control. 63 (2018) 2465. http://doi. org/10.1109/TAC.2017.2771336 